\title{
Anaphylactic reaction with hydroxyethyl starch during anesthesia \\ - A case report -
}

Received September 13, 2018

Revised 1st, December 18, 2018

2nd, December 27, 2018

Accepted March 5, 2019

\section{Corresponding author}

Mijung Yun, M.D., Ph.D.

Department of Anesthesiology and

Pain Medicine, National Medical

Center, 245 Eulji-ro, Jung-gu, Seoul

04564, Korea

Tel: 82-2-2260-7370

Fax: 82-2-2262-4766

E-mail: mijung.yun@nmc.or.kr

\section{ORCID}

https://orcid.org/0000-0001-5838-4893

\section{Gunnhee Kim, Goeun Kim, Miyoung Kwon, Minseok Koo, and Mijung Yun}

Department of Anesthesiology and Pain Medicine, National Medical Center, Seoul, Korea
Background: Hydroxyethyl starch (HES), a class of synthetic colloid solutions, has been widely used to treat perioperative hypovolemia. The use of HES, however, is associated with the risk of allergic reactions.

Case: An 83-year-old man was scheduled to undergo an open reduction and internal fixation of a pertrochanteric fracture under spinal anesthesia. He had no history of allergy. Five minutes after HES administration, hypotension, agitation, and skin rash were developed. HES infusion was terminated due to a suspected anaphylactic reaction. The vital signs recovered following administration of phenylephrine, dexamethasone, and hydrocortisone. Serum tryptase and total immunoglobulin E levels were elevated in plasma samples collected following the commencement of the allergic reaction during surgery.

Conclusions: In the present report, the risk of anaphylactic reaction with HES and the laboratory tests needed to support the diagnosis are highlighted.

Keywords: Anaphylaxis; Anesthesia, Spinal; Starch.
Hydroxyethyl starch (HES) is a colloid commonly used to treat perioperative hypovolemia. HES belongs to a class of synthetic colloid solutions that are modified natural polysaccharides and are similar to glycogen [1]. The physicochemical characteristics of HES are determined by the concentration, mean molecular weight $\left(\mathrm{M}_{\mathrm{W}}\right)$, degree of molar substitution (MS), and $\mathrm{C}_{2} / \mathrm{C}_{6}$ ratio.

The risk of an anaphylactic reaction, which may be a side effect of HES, is very low [2]. In a clinical trial, the use of HES was associated with a low incidence of anaphylactic reactions, similar to that observed with the use of albumin. This risk was lower than that associated with the use of other colloids [3]. As the allergic reaction appears to be induced by the substance itself (starch), all generations of HES may exhibit anaphylactic potency. There have been several cases of ana- phylactic reaction induced by first- and second-generation HES and only one case of such a reaction with the third-generation HES. The HES used in the present case was balanced tetrastarch, which is a third-generation HES. We present a patient who developed an intra-operative anaphylactic reaction immediately after the initiation of HES 130/0.4 infusion, along with a review of the literature.

\section{CASE REPORT}

An 83-year-old man (weight: $65 \mathrm{~kg}$, height: $167 \mathrm{~cm}$ ) was scheduled to undergo open reduction and internal fixation of a pertrochanteric fracture of the right femur. His past medical history was nonspecific. He had no history of allergy to drugs or food. Preoperative assessment including blood biochem-

This is an Open Access article distributed under the terms of the Creative Commons Attribution Non-Commercial License (http://creativecommons.org/licenses/by-nc/4.0) which permits unrestricted non-commercial use, distribution, and reproduction in any medium, provided the original work is properly cited.

Copyright (C) the Korean Society of Anesthesiologists, 2019 
istry, chest X-ray, and electrocardiography showed results within the normal range. Two hours before arriving at the operating room, the patient was administered $1 \mathrm{~g}$ of cefotetan sodium (Yamatetan ${ }^{\circledR}$, Jeilpharm, Korea) intravenously (IV). Preoperative skin test to the Yamatetan ${ }^{\circledR}$ was negative.

Upon arriving at the operating room, the patient was monitored using noninvasive blood pressure (BP) measurement, electrocardiography, and pulse oximetry $\left(\mathrm{SpO}_{2}\right)$ equipment. The pre-anesthetic $\mathrm{BP}$, heart rate (HR), and $\mathrm{SpO}_{2}$ values were 172/108 $\mathrm{mmHg}$, 94 beats/min, and 95\%, respectively.

Spinal anesthesia was administered in the left lateral decubitus position using hyperbaric bupivacaine ( $13 \mathrm{mg}$ ) and fentanyl $(10 \mu \mathrm{g})$ while oxygen was supplied at $6 \mathrm{~L} / \mathrm{min}$ via a facial mask. After 5 min, sensory blockade of T10 was checked. For sedation, $1 \mathrm{mg}$ of IV midazolam was administered. During the $50 \mathrm{~min}$ of the spinal anesthesia, that is $25 \mathrm{~min}$ after the start of surgery, the systolic blood pressure was between 110 and 120 and pulse rate was maintained in the early $100 \mathrm{~s}$, and these were stable. By then, the patient had received 0.4-0.5 L of crystalloid (plasma solution), the estimated blood loss was about $50 \mathrm{ml}$, and the urine output was about $1.5 \mathrm{ml} / \mathrm{kg} / \mathrm{h}$.

After $25 \mathrm{~min}$ from the start of surgery, $\mathrm{BP}, \mathrm{HR}, \mathrm{SpO}_{2}$ values were 116/72 $\mathrm{mmHg}$, 94 beats/min and 100\%, respectively. The HES 130/0.4 (6\% Volulyte ${ }^{\circledR}$, Fresenius Kabi Korea, Korea) infusion was started through a peripheral IV route to replace the expected post-operative bone bleeding and an additional $2 \mathrm{mg}$ of IV midazolam was administered. Five minutes after the administration of $50 \mathrm{ml}$ of HES, the BP suddenly dropped to $84 / 52 \mathrm{mmHg}$, and the HR increased to 111 beats $/ \mathrm{min}$. $\mathrm{SpO}_{2}$ was reduced to $95 \%$. To restore the BP, $100 \mu \mathrm{g}$ of IV phenylephrine was administered immediately. Subsequently, the patient exhibited confusion and agitation. For further sedation, $1 \mathrm{mg}$ of midazolam was administered, but the patient did not calm down and tried to move his fixed arms. Skin rash was observed on the face and neck first, which then spread to the entire body. The rechecked BP, $\mathrm{HR}$, and $\mathrm{SpO}_{2}$ after phenylephrine administration, were 84/45 mmHg, 117 beats/min, and $97 \%$, respectively. Additional $100 \mu \mathrm{g}$ of IV phenylephrine bolus was administered. Immediately, the test for skin sensitivity to Yamatetan ${ }^{\circledR}$ was re-performed, and the result was negative. The patient's agitation was reduced and the vital signs were maintained after multiple phenylephrine bolus were administered. The surgery was nearing completion; hence, general anesthesia was not considered.
Clinical signs including hypotension, tachycardia, and mild hypoxemia, as well as the appearance of a skin rash were consistent with the clinical criteria for an anaphylactic reaction. Other antibiotics or blood components were not administered during the surgery, and the anaphylactic reaction occurred within 5 min of HES exposure. The infusion of HES was terminated within $10 \mathrm{~min}$, as it was considered the cause of the anaphylactic reaction. Subsequently, $5 \mathrm{mg}$ of IV dexamethasone was administered. The total amount of IV injected phenylephrine was $400 \mu \mathrm{g}$. The patient received $1 \mathrm{~L}$ of the crystalloid (plasma solution) and $50 \mathrm{ml}$ of the colloid (HES). Estimated blood loss and urine output were 100 $\mathrm{ml}$ and $200 \mathrm{ml}$, respectively. The total anesthesia time was 2 hours and the operation time was $46 \mathrm{~min}$.

In the recovery room, laboratory tests including immunoglobulin E (IgE) levels and tryptase assessment were performed. The patient was taken chest $\mathrm{X}$-ray due to observation of mild hypoxemia; however, the radiologist interpreted no unusual findings compared with the preoperative examination. The skin rash on the entire body persisted while the patient stayed in the recovery room; hence, IV dexamethasone $7.5 \mathrm{mg}$ and chlorpheniramine $6 \mathrm{mg}$ were administered along with $800 \mathrm{ml}$ of crystalloid fluid for symptom relief.

The patient was transferred to the general ward with stable vital signs (BP: 111/78 mmHg, HR: 96 beats/min, $\mathrm{SpO}_{2}$ : 100\%) after close observation in the recovery room for $75 \mathrm{~min}$. The patient's urine output was sufficient $(1-2 \mathrm{ml} / \mathrm{kg} / \mathrm{h})$ for $24 \mathrm{~h}$ after operation.

The result of the skin test to detect a reaction against HES performed on the next day was negative. Testing of a blood sample drawn in the operating room revealed the presence of $13.9 \mathrm{ng} / \mathrm{ml}$ of tryptase (normal range: $<11.4 \mathrm{ng} / \mathrm{ml}$ ) and 211 $\mathrm{IU} / \mathrm{ml}$ of total IgE, as determined using paper radioimmunosorbent test (PRIST) (normal range: $0-100 \mathrm{IU} / \mathrm{ml}$ ). On the twenty-first day after the surgery, the patient was discharged without any complications.

\section{DISCUSSION}

HES is derived from amylopectin of maize or potato. HES solutions can be classified by their $\mathrm{M}_{\mathrm{w}}$ or MS. Volulyte is a third generation HES with balanced $130\left(\mathrm{M}_{\mathrm{w}}, \mathrm{kDa}\right) / 0.4$ (MS) or $130\left(\mathrm{M}_{\mathrm{w}}, \mathrm{kDa}\right) / 0.42(\mathrm{MS})$.

An anaphylactic reaction is defined as a serious allergic 
reaction that is rapid in onset and may cause death [4]. Symptoms are variable, ranging from minor clinical changes such as agitation, urticaria to cardiopulmonary collapse.

The diagnosis of an anaphylactic reaction is usually obvious and is made based on the symptoms. If the symptoms are mild, the diagnosis can be confirmed using a blood or urine test, which provide a measure the levels of substances produced during an allergic reaction [1]. Traditionally, in addition to the clinical features, serum tryptase, plasma histamine, and 24-h urinary histamine metabolites have been clinically used to confirm the diagnosis of anaphylactic reactions. Skin tests and allergen-specific IgE tests can provide confirmatory evidence of sensitization to a specific allergen.

In the present case, severe and widespread skin rash and agitation were the first signs of the reaction. Tachypnea, mild hypoxemia, and hypotension were the subsequent signs that confirmed the suspicion of an anaphylactic reaction. All of the clinical signs were detected within a few minutes after the infusion of HES. Fortunately, bronchospasm did not develop in the current case.

Treatment of an anaphylactic reaction consists of both short and long-term management. The immediate goal is to maintain an airway with oxygen supply and support the blood pressure. Intravenous epinephrine diluted with $1: 10,000$ is administered slowly, the dose is usually limited to $3 \mathrm{ml}$ for an average sized (70 kg) adult. Hypotension should be treated by expanding the intravascular volume. When a prolonged course with urticarial or a late phase response is suspected, antihistamines and corticosteroids are useful as second line therapy. Methylprednisolone sodium succinate is usually given every $6 \mathrm{~h}$. Inhaled beta 2 agonist and intravenous atropine are also recommended for refractory bronchospasm.

In the present case, a skin test for a reaction against HES was performed on the next day of the surgery. The result of this test was negative. High doses of systemic corticosteroids and antihistamines may interfere with the results of skin tests, and it is important to avoid these drugs for up to 7 days before the test [5]. We had already administered an antihistamine and corticosteroids due to the allergic reaction. The administration of these drugs may thus have induced the negative results. The elevated values of total IgE and tryptase measured during the surgery support the diagnosis of an anaphylactic reaction due to HES. One day after the surgery, the patient was delirious and uncooperative. The patient's family refused additional tests for the patient because of the difficulty of nursing and old age of the patient. They thought that the patient would not have another surgery in the future due to his old age. Therefore, the confirmatory tests such as plasma histamine, 24-h urinary histamine metabolites, and allergenspecific IgE evaluations could not be performed.

There have been several case reports of anaphylactic reactions against HES. However, a majority of these reactions were against $6 \%$ dextran 60 [6] and first-generation (hetastarch) [7-10], or second-generation (pentastarch) [11-13] HES. Only one case involving a third-generation HES (tetrastarch)-induced anaphylactic reaction has been reported. A 42-year-old man experienced a severe intra-operative anaphylactic reaction soon after the initiation of HES 130/0.4 (Voluven $^{\circledR}$, Fresenius Kabi, Germany) infusion. Within minutes of HES administration, facial erythema, hypotension, and bronchospasm developed, however the measurement of serum tryptase, plasma histamine, or allergen-specific IgE or skin tests were not performed [14].

All colloids used for intravascular volume replacement, including the natural colloid albumin, have the potential to induce anaphylactic reactions [15]. In a large clinical trial that included approximately 20,000 patients, it was demonstrated that the use of HES was associated with a low incidence of anaphylactic reactions similar to that with albumin, and significantly less than that with other colloids [3]. Data on the incidence of anaphylactic reactions after IV administration of the HES 130/0.4 are not available.

Fortunately, anaphylactic reactions induced by HES are uncommon, and life-threatening anaphylaxis is rare. The diagnosis of anaphylactic reaction could be difficult while the patient is under anesthesia and when various types of medications are used simultaneously. Therefore, it is essential to know the past medical history of the patient's allergic reactions, be aware of the criteria for the diagnosis, and provide prompt treatment for anaphylactic reactions.

\section{CONFLICTS OF INTEREST}

No potential conflict of interest relevant to this article was reported. 


\section{ORCID}

Gunnhee Kim: https://orcid.org/0000-0002-0014-8297

Goeun Kim: https://orcid.org/0000-0002-7223-6317

Miyoung Kwon: https://orcid.org/0000-0002-0667-9494

Minseok Koo: https://orcid.org/0000-0002-2012-9616

\section{REFERENCES}

1. Jungheinrich C, Neff TA. Pharmacokinetics of hydroxyethyl starch. Clin Pharmacokinet 2005; 44: 681-99.

2. Boldt J. Modern rapidly degradable hydroxyethyl starches: current concepts. Anesth Analg 2009; 108: 1574-82.

3. Laxenaire MC, Charpentier C, Feldman L. Anaphylactoid reactions to colloid plasma substitutes: incidence, risk factors, mechanisms. A French multicenter prospective study. Ann Fr Anesth Reanim 1994; 13: 301-10.

4. Lieberman P, Nicklas RA, Randolph C, Oppenheimer J, Bernstein $\mathrm{D}$, Bernstein J, et al. Anaphylaxis--a practice parameter update 2015. Ann Allergy Asthma Immunol 2015; 115: 341-84.

5. Kränke B, Aberer W. Skin testing for IgE-mediated drug allergy. Immunol Allergy Clin North Am 2009; 29: 503-16.

6. Lehmann G, Asskali F, Förster H. Severe adverse event following iv administration of $10 \mathrm{ml}$ 6\% Dextran60 (0.6 g) in a healthy volunteer. Anaesthesist 2002; 51: 820-4.

7. Takada M, Tomatsu T, Harada T, Murakami N, Shimonaka H,
Dohi S. Bronchospasm due to anaphylactic reaction to Hydroxyethyl starch (HESPANDER). Masui 1997; 46: 397-400.

8. Hall BA, Frigas E, Matesic D, Gillett MD, Sprung J. Case report: intraoperative anaphylactoid reaction and hydroxyethyl starch in balanced electrolyte solution (Hextend). Can J Anaesth 2006; 53: 989-93.

9. Cullen MJ, Singer M. Severe anaphylactoid reaction to hydroxyethyl starch. Anaesthesia 1990; 45: 1041-2.

10. Porter SS, Goldberg RJ. Intraoperative allergic reactions to hydroxyethyl starch: a report of two cases. Can Anaesth Soc J 1986; 33(3 Pt 1): 394-8.

11. Vercauteren MP, Coppejans HC, Sermeus L. Anaphylactoid reaction to hydroxyethylstarch during cesarean delivery in a patient with HELLP syndrome. Anesth Analg 2003; 96: 859-61.

12. Kannan S, Milligan KR. Moderately severe anaphylactoid reaction to pentastarch $(200 / 0.5)$ in a patient with acute severe asthma. Intensive Care Med 1999; 25: 220-2.

13. McHugh GJ. Anaphylactoid reaction to pentastarch. Can J Anaesth 1998; 45: 270-2.

14. Kim HJ, Kim SY, Oh MJ, Kim JM. Anaphylaxis induced by hydroxyethyl starch during general anesthesia -a case report-. Korean J Anesthesiol 2012; 63: 260-2.

15. Franz A, Bräunlich P, Gamsjäger T, Felfernig M, Gustorff B, Kozek-Langenecker SA. The effects of hydroxyethyl starches of varying molecular weights on platelet function. Anesth Analg 2001; 92: 1402-7. 